\title{
FILTERED PERVERSE COMPLEXES
}

\author{
Paul Bressler, Morihiko Saito, and Boris Youssin
}

\begin{abstract}
We introduce the notion of filtered perversity of a filtered differential complex on a complex analytic manifold $X$, without any assumptions of coherence, with the purpose of studying the connection between the pure Hodge modules and the $L^{2}$-complexes.

We show that if a filtered differential complex $\left(\mathcal{M}^{\bullet}, F_{\bullet}\right)$ is filtered perverse then $\mathrm{DR}^{-1}\left(\mathcal{M}^{\bullet}, F_{\bullet}\right)$ is isomorphic to a filtered $\mathcal{D}$-module; a coherence assumption on the cohomology of $\left(\mathcal{M}^{\bullet}, F_{\bullet}\right)$ implies that, in addition, this $\mathcal{D}$-module is holonomic.

We show the converse: the de Rham complex of a holonomic Cohen-Macaulay filtered $\mathcal{D}$-module is filtered perverse.
\end{abstract}

\section{Introduction}

1.1. Cheeger-Goresky-MacPherson conjectures. J. Cheeger, M. Goresky and R. MacPherson [CGM] conjectured some fifteen years ago that the intersection cohomology of a singular complex projective algebraic variety is naturally isomorphic to its $L^{2}$ cohomology and the Kähler package holds for them. Their motivation was as follows.

The intersection cohomology was discovered by M. Goresky and R. MacPherson [GM1], [GM2] as an invariant of stratified spaces which for complex algebraic varieties might serve as a replacement of the usual cohomology: it had some properties that the usual cohomology of smooth projective varieties possessed but the usual cohomology of singular projective varieties did not. One of such properties was Poincaré duality which is a part of the "Kähler package" of properties that hold in the smooth case.

At the same time, J. Cheeger discovered that the $L^{2}$ cohomology groups of varieties with conical singularities have properties similar to those of intersection cohomology, and he proved in this case the Hodge-de Rham isomorphism between the $L^{2}$ cohomology that he defined and studied, and the intersection cohomology $[\mathrm{C}]$.

The hope that underlied these conjectures was that it would be possible to use the $L^{2}$ Kähler methods to prove the Kähler package for intersection cohomology similarly to the way the Kähler package was proved for the usual cohomology of complex projective manifolds.

Received September 22, 1997.

The last author was partially supported by NSF grants at MIT and Indiana University, Bloomington 
The most important part of the Kähler package is the $(p, q)$-decomposition in the cohomology groups (the "Hodge structure").

The definition of $L^{2}$ cohomology involves a metric (Riemannian or Kähler) defined almost everywhere on the variety (e.g. on its nonsingular part). The most important metric comes from a projective embedding of the variety and is induced by the Fubini-Studi metric on the projective space. (The $L^{2}$ cohomology is independent of the choice of the imbedding.)

The isomorphism with intersection cohomology is known in case of surfaces $[\mathrm{HP}]$, [N1] and in case of isolated singularities of any dimension both for FubiniStudi metric [O1], [O2] and for a different, complete metric, introduced by L. Saper, which is defined on the nonsingular part of the variety and blows up near the singularities [Sap]. The $(p, q)$-decomposition is known for the case of Fubini-Studi metrics only in cases of dimension two [N2] (except for the middle degree cohomology groups) while a classical result of Andreotti-Vesentini implies the $(p, q)$-decomposition for any complete metric. The general case is still open, despite the announcement of T. Ohsawa [O3].

In the meantime the second author [S1], [S2] developed a theory of polarizable Hodge modules which implied the Kähler package for the intersection cohomology. His main tool was the theory of $\mathcal{D}$-modules and his methods were essentially algebraic, reducing the intersection cohomology to the intersection cohomology of a curve with coefficients in a polarised variation of Hodge structure [Z1].

1.2. The comparison between the Hodge structures. Assuming that the Cheeger-Goresky-MacPherson conjectures are true, one is faced with the question of comparison between the two Hodge structures on the intersection cohomology: one induced by the isomorphism with $L^{2}$ cohomology, the other coming from the theory of polarised Hodge modules. In fact, different metrics give different $L^{2}$ cohomology theories and hence, pose different comparison problems.

In case of isolated singularities, S. Zucker [Z2] proved the coincidence between the Hodge structures coming from polarized Hodge modules and from $L^{2}$ cohomology with respect to the Saper metric (or arithmetic quotient metrics similar to it). Some partial results are also known in case of Fubini-Studi metric, see $[\mathrm{Z} 2]$ and [N2].

It is interesting to note that the original purpose of the conjectures was to construct the Hodge structure on the intersection cohomology. The $L^{2}$ methods, however, turned out to be so difficult that the Hodge structure was constructed by different, algebraic methods and now we are faced with the problem of comparison between the two Hodge structures.

1.3. The local comparison problem. A major component of a polarizable Hodge module is a regular holonomic $\mathcal{D}$-module $M$ with a good filtration $F_{\bullet}$. Suppose $M$ corresponds to the intersection cohomology complex of a complex projective subvariety $Z$; the correspondence is given by taking the de Rham complex $\operatorname{DR}(M)$ of $M$ (so that $\operatorname{DR}(M)$ is isomorphic to the intersection coho- 
mology complex of $Z$ ). Then the filtration $F_{\text {• }}$ induces a filtration on $\operatorname{DR}(M)$ which yields the Hodge structure on the intersection cohomology.

The complex $\operatorname{DR}\left(M, F_{\bullet}\right)$ is a filtered differential complex [S1]: a complex of sheaves which are modules over the sheaf of analytic functions and the differentials are differential operators. This filtered differential complex completely determines $\left(M, F_{\bullet}\right)$ as there is an inverse functor $\mathrm{DR}^{-1}[\mathrm{~S} 1]$.

If the metric used in the construction of the $L^{2}$ complex (it is a Kähler metric on the nonsingular part of $Z$ ) is bounded below with respect to Fubini-Studi metric, the $L^{2}$ complex is a filtered differential complex (see, e.g., 6.1 below).

The local comparison problem is as follows: is it true that the de Rham complex of the Hodge module is isomorphic to the $L^{2}$ complex in the derived category of filtered differential complexes?

The intersection cohomology can be taken with coefficients in a local system defined on the non-singular part of $Z$ or a Zariski-open subset of it. If this local system underlies a polarized variation of Hodge structures then a corresponding polarized Hodge module can be constructed and the intersection cohomology with coefficients in this local system has a Hodge structure. On the other hand, the $L^{2}$ cohomology can be taken with coefficients in the same polarized variation, and we can ask the same local comparison question in this situation.

1.4. Weak filtered perversity. A way to approach this problem is to try to identify the properties of a filtered differential complex $\left(\mathcal{M}^{\bullet}, F_{\bullet}\right)$ which would imply that $\mathrm{DR}^{-1}\left(\mathcal{M}^{\bullet}, F_{\bullet}\right)$ is isomorphic to the filtered $\mathcal{D}$-module which underlies a polarized Hodge module.

In general, $\mathrm{DR}^{-1}\left(\mathcal{M}^{\bullet}, F_{\bullet}\right)$ is a complex of filtered $\mathcal{D}$-modules; in this paper we study properties of $\left(\mathcal{M}^{\bullet}, F_{\bullet}\right)$ which imply that this complex is isomorphic to one filtered $\mathcal{D}$-module in the filtered derived category.

We call these properties weak filtered perversity (see Definition 3.2.1); it means that, first, the complex $\left(\mathcal{M}^{\bullet}, F_{\bullet}\right)$ is locally trivial along the strata - in a certain filtered sense - with respect to some analytic stratification, and second, it satisfies certain local filtered cohomology vanishing which is similar to the local cohomology vanishing of perverse sheaves. No coherence assumption is being made on $\left(\mathcal{M}^{\bullet}, F_{\bullet}\right)$.

In case $\left(\mathcal{M}^{\bullet}, F_{\bullet}\right)$ is the $L^{2}$ complex, the cohomology that must vanish, turn out to be a version of the $L^{2}$ - $\bar{\partial}$-cohomology, see $\S 6.2$ below for the discussion.

1.5. The main results. We show (see Theorem 4.1.1) that if $\left(\mathcal{M}^{\bullet}, F_{\bullet}\right)$ is weakly filtered perverse then, indeed, $\mathrm{DR}^{-1}\left(\mathcal{M}^{\bullet}, F_{\bullet}\right)$ is isomorphic to a filtered $\mathcal{D}$-module.

We show the converse (see Theorem 5.3.1): if $\left(M^{\bullet}, F_{\bullet}\right)$ is a coherent filtered $\mathcal{D}$-module which is Cohen-Macaulay (i.e., its dual in the filtered sense is also a complex of filtered $\mathcal{D}$-modules isomorphic to one filtered $\mathcal{D}$-module) then $\operatorname{DR}\left(M, F_{\bullet}\right)$ is weakly filtered perverse. 
We show (see Proposition 5.2.1) that a coherence assumption together with filtered perversity of $\left(\mathcal{M}^{\bullet}, F_{\bullet}\right)$ implies that the filtered $\mathcal{D}$-module $\operatorname{DR}^{-1}\left(\mathcal{M}^{\bullet}, F_{\bullet}\right)$ is holonomic.

1.6. Plan of the paper. In Section 2 we review the necessary background material from [S1].

In Section 3 we introduce the notion of weak filtered perversity.

In Sections 4 and 5 we prove the results listed above.

In Section 6 we give a modest application: we strengthen the results of [KK2] and [S1] and show (in the situation of [KK2]) that filtered perversity of the $L^{2}$ complex implies the local filtered isomorphism (in the sense of derived category) between the $L^{2}$ complex and the de Rham complex of the $\mathcal{D}$-module that underlies the corresponding pure Hodge module.

\section{Filtered $\mathcal{D}$-modules and differential complexes}

In this section we make a brief survey of the necessary parts of [S1].

2.1. General notation. Throughout this paper $X$ will denote a complex manifold, $\mathcal{O}_{X}$ the sheaf of holomorphic functions, $\omega_{X}$ the canonical sheaf of $X, \mathcal{D}_{X}$ the sheaf of differential operators. Unless specified otherwise, a $\mathcal{D}_{X}$-module will always refer to a sheaf of right modules over $\mathcal{D}_{X}$.

For a complex of sheaves $\mathcal{F}^{\bullet}$, we shall denote by $H^{j} \mathcal{F}^{\bullet}$ its sheaf cohomology, and by $H_{\{x\}}^{j} \mathcal{F}^{\bullet}$ its (hyper)cohomology with supports in a one-point set $\{x\}$.

2.2. Filtered $\mathcal{D}$-modules. Recall that $\mathcal{D}_{X}$ is a filtered ring when equipped with the filtration $F_{\bullet} \mathcal{D}_{X}$, where $F_{p} \mathcal{D}_{X}$ is the $\mathcal{O}_{X}$-module of $\mathcal{D}_{X}$ of operators of order at most $p$.

A filtered $\mathcal{D}_{X}$-module is a pair $\left(M, F_{\bullet}\right)$ consisting of a $\mathcal{D}_{X}$-module $M$ and a filtration $F_{\bullet} M$ of $M$ by $\mathcal{O}_{X}$ submodules compatible with the action of $\mathcal{D}_{X}$ and the filtration on the latter.

We refer the reader to [S1], $\S 2.1$ for the precise definition of the category of filtered $\mathcal{D}_{X}$-modules and its derived category, in various flavors; what is important for us now, is that the derived category of filtered $\mathcal{D}_{X}$-modules is isomorphic to the derived category of the category whose objects are filtered $\mathcal{O}_{X}$-modules and whose morphisms are differential operators that agree with the filtration in a certain way.

This equivalence is given by the two functors, $\mathrm{DR}_{X}$ and $\mathrm{DR}_{X}^{-1}$ (loc. cit.) which act as follows.

2.3. The de Rham functor $\mathrm{DR}_{X}$. For a filtered $\mathcal{D}_{X}$-module $\left(M, F_{\bullet}\right)$, the filtered differential complex $\operatorname{DR}_{X}\left(M, F_{\bullet}\right)$ is the usual de Rham complex of $M$, given by

$$
\operatorname{DR}_{X} M=M \otimes_{\mathcal{D}_{X}}\left(\mathcal{D}_{X} \otimes_{\mathcal{O}_{X}} \Lambda^{-\bullet} \Theta_{X}\right)=M \otimes_{\mathcal{O}_{X}} \Lambda^{-\bullet} \Theta_{X}
$$


where $\Theta_{X}$ is the tangent sheaf to $X, \Lambda^{-\bullet} \Theta_{X}$ its exterior algebra with $p$-th exterior power placed in degree $-p$, the differential is given by

$$
\begin{aligned}
& d\left(P \otimes \xi_{1} \wedge \ldots \wedge \xi_{p}\right)=\sum_{i=1}^{p}(-1)^{i-1} P \xi_{i} \otimes \xi_{1} \wedge \ldots \wedge \widehat{\xi}_{i} \wedge \ldots \wedge \xi_{p} \\
&+\sum_{1 \leq i<j \leq p}(-1)^{i+j} P \otimes\left[\xi_{i}, \xi_{j}\right] \wedge \xi_{1} \wedge \ldots \wedge \widehat{\xi}_{i} \wedge \ldots \wedge \widehat{\xi}_{j} \wedge \ldots \wedge \xi_{p}
\end{aligned}
$$

(it corresponds to the differential in $\mathcal{D}_{X} \otimes_{\mathcal{O}_{X}} \bigwedge^{-\bullet} \Theta_{X}$ which makes it the standard Koszul resolution of $\mathcal{O}_{X}$ as a $\mathcal{D}_{X}$-module), and the filtration on $\mathrm{DR}_{X} M$ is given by

$$
F_{p}\left(M \otimes_{\mathcal{O}_{X}} \bigwedge^{-i} \Theta_{X}\right)=F_{p+i} M \otimes_{\mathcal{O}_{X}} \bigwedge^{-i} \Theta_{X}
$$

For a complex of filtered $\mathcal{D}_{X}$-modules $\left(M^{\bullet}, F_{\bullet}\right)$, the filtered differential complex $\operatorname{DR}_{X}\left(M^{\bullet}, F_{\bullet}\right)$ is the total complex of $\operatorname{DR}_{X}\left(M^{q}, F_{\bullet}\right)$ for all $q$.

(Note that what we denote by $\mathrm{DR}_{X}$, was denoted by $\widetilde{\mathrm{DR}}$ in $[\mathrm{S} 1]$. )

2.4. The inverse de $R$ ham functor $D R_{X}^{-1}$. For a filtered differential complex $\left(\mathcal{M}^{\bullet}, F_{\bullet}\right)$, the complex of filtered $\mathcal{D}_{X}$-modules $\operatorname{DR}_{X}^{-1}\left(\mathcal{M}^{\bullet}, F_{\bullet}\right)$ is described as the complex of differential operators from $\mathcal{O}_{X}$ into $\left(\mathcal{M}^{\bullet}, F_{\bullet}\right)$ with the obvious differential and filtration. The action of $\mathcal{D}_{X}$, i. e., differential operators $\mathcal{O}_{X} \rightarrow$ $\mathcal{O}_{X}$, is by composition. The individual terms of this complex are simply $\mathcal{M}^{j} \otimes_{\mathcal{O}_{X}}$ $\mathcal{D}_{X}$.

The two functors $\mathrm{DR}_{X}$ and $\mathrm{DR}_{X}^{-1}$ are inverse to each other in the derived categories.

2.5. Duality. For a bounded complex of filtered $\mathcal{D}_{X}$-modules $\left(M^{\bullet}, F_{\bullet}\right)$, its dual $\mathbb{D}\left(M, F_{\bullet}\right)$ is another complex of filtered $\mathcal{D}_{X}$-modules defined ([S1], 2.4.3) in such way that it agrees with various other duality functors, as follows.

There is a duality functor (also denoted by $\mathbb{D}$ ) on filtered differential complexes which on an individual $\mathcal{O}_{X}$-module $L$ is defined as $\mathbb{D}(L)=\mathbf{R} \underline{\operatorname{Hom}}_{\mathcal{O}_{X}}\left(L, \omega_{X}[n]\right)$ where $n=\operatorname{dim} X$ ([S1], 2.4.11), and such that in the appropriate derived categories the functors $\mathbb{D} \circ \mathrm{DR}_{X}$ and $\mathrm{DR}_{X} \circ \mathbb{D}$ are isomorphic.

For a filtered differential complex $\left(\mathcal{M}^{\bullet}, F_{\bullet}\right)$ we have

$$
\operatorname{Gr}_{\bullet}^{F} \mathbb{D}\left(\mathcal{M}^{\bullet}, F_{\bullet}\right) \cong \mathbf{R} \underline{\operatorname{Hom}}_{\mathcal{O}_{X}}\left(\operatorname{Gr}_{\bullet}^{F} \mathcal{M}^{\bullet}, \omega_{X}[n]\right)
$$

In case $\left(M^{\bullet}, F_{\bullet}\right)$ is a complex of filtered coherent $\mathcal{D}_{X}$-modules (i.e., coherent $\mathcal{D}_{X}$-modules with good filtrations), the complex of $\mathcal{D}_{X}$-modules which underlies $\mathbb{D}\left(M^{\bullet}, F_{\bullet}\right)$, is the usual dual of $M^{\bullet}$. In addition, under the same assumptions $\mathbb{D D}\left(M^{\bullet}, F_{\bullet}\right) \cong\left(M^{\bullet}, F_{\bullet}\right)$. 
2.6. Restriction to a noncharacteristic submanifold. Let $Y$ be a smooth submanifold of codimension $d$ in $X$; denote the embedding $i: Y \hookrightarrow X$ and $\omega_{Y / X}=\omega_{Y} \otimes_{\mathcal{O}_{X}} \omega_{X}^{-1}$.

We say that a bounded complex of filtered $\mathcal{D}_{X}$-modules $\left(M^{\bullet}, F_{\bullet}\right)$ is weakly noncharacteristic with respect to $Y$ (or $Y$ with respect to $\left(M^{\bullet}, F_{\bullet}\right)$ ) if it satisfies the property

$$
\underline{\operatorname{Tor}}_{k}^{\mathcal{O}_{X}}\left(H^{j}\left(\operatorname{Gr}_{p}^{F} M^{\bullet}\right), \mathcal{O}_{Y}\right)=0 \quad \text { for all } k \neq 0, j \text { and } p .
$$

Under this assumption, the noncharacteristic restriction $\left(M^{\bullet}, F_{\bullet}\right)_{Y}$ is defined as follows:

$$
\left(M^{\bullet}, F_{\bullet}\right)_{Y}=\left(M^{\bullet}, F_{\bullet}\right) \otimes_{\mathcal{D}_{X}}^{\mathbb{L}}\left(\mathcal{D}_{X \leftarrow Y}, F_{\bullet}\right)
$$

where $\mathcal{D}_{X \leftarrow Y}=\mathcal{D}_{X} \otimes_{\mathcal{O}_{X}} \omega_{Y / X}$ is the usual $\left(\mathcal{D}_{X}, \mathcal{D}_{Y}\right)$-bimodule with the filtration $F_{p} \mathcal{D}_{X \leftarrow Y}=F_{p-d} \mathcal{D}_{X} \otimes_{\mathcal{O}_{X}} \omega_{Y / X}$; the restriction $\left(M^{\bullet}, F_{\bullet}\right)_{Y}$ thus defined, is a complex of right $\mathcal{D}_{Y}$-modules. As a complex of $\mathcal{O}_{Y}$-modules, it can be described as $\left(M^{\bullet}\right)_{Y}=M^{\bullet} \otimes_{\mathcal{O}_{X}}^{\mathbb{L}} \omega_{Y / X}$ with the filtration $F_{p}\left(M^{\bullet}\right)_{Y}=F_{p-d} M^{\bullet} \otimes_{\mathcal{O}_{X}}^{\mathbb{L}} \omega_{Y / X}$. We have

$$
H^{j} \operatorname{Gr}_{p}^{F}\left(\left(M^{\bullet}, F_{\bullet}\right)_{Y}\right) \stackrel{\simeq}{=} H^{j}\left(\operatorname{Gr}_{p-d}^{F} M^{\bullet}\right) \otimes_{\mathcal{O}_{X}} \omega_{Y / X}
$$

Suppose that $\left(M^{\bullet}, F_{\bullet}\right)$ has the property that the complex $\operatorname{Gr}_{\bullet}^{F} M^{\bullet}$ has bounded $\mathrm{Gr}_{\bullet}^{F} \mathcal{D}_{X}$-coherent cohomology; in such case we say that $\left(M^{\bullet}, F_{\bullet}\right)$ is noncharacteristic with respect to $Y$ if, first, $(2.2)$ is satisfied, and second, $\operatorname{Gr}_{\bullet}^{F}\left(M^{\bullet}\right)_{Y}$ also has bounded $\mathrm{Gr}^{F} \mathcal{D}_{Y}$-coherent cohomology.

In the particular case when the complex $\left(M^{\bullet}, F_{\bullet}\right)$ is actually a filtered coherent $\mathcal{D}_{X}$-module $\left(M, F_{\bullet}\right)$, this definition is equivalent to the definition in [S1], 3.5.1 because the condition of coherence of $\left(M^{\bullet}, F_{\bullet}\right)_{Y}$ is is equivalent to the finiteness of the projection $\left(Y \times_{X} T^{*} X\right) \cap \mathrm{Ch}(M) \rightarrow T^{*} Y$ where $\mathrm{Ch}(M)$ denotes the characteristic variety of $M$. In such case if $Y$ is noncharacteristic, we have $i^{*}\left(M, F_{\bullet}\right)=\left(M^{\bullet}, F_{\bullet}\right)_{Y}[d]$ and $i^{!}\left(M, F_{\bullet}\right)$ is isomorphic to $\left(M^{\bullet}, F_{\bullet}\right)_{Y}[-d]$ up to a shift of filtration.

Definition 2.6.1. A filtered coherent $\mathcal{D}_{X}$-module $\left(M, F_{\bullet}\right)$ is Cohen-Macaulay if $\operatorname{Gr}_{\bullet}^{F} M$ is a Cohen-Macaulay module over $\operatorname{Gr}_{\bullet}^{F} \mathcal{D}$.

A Cohen-Macaulay $\mathcal{D}_{X}$-module $\left(M, F_{\bullet}\right)$ is holonomic iff the dimension of $\operatorname{Gr}_{\bullet}^{F} M$ over $\operatorname{Gr}_{\bullet}^{F} \mathcal{D}$ is equal to $\operatorname{dim} X$.

Lemma 2.6.2. Suppose that $\left(M, F_{\bullet}\right)$ is a coherent holonomic filtered $\mathcal{D}_{X}$-module noncharacteristic with respect to $Y$. Then $\left(M, F_{\bullet}\right)$ is holonomic Cohen-Macaulay at a point $y \in Y$ if and only if $\left(M, F_{\bullet}\right)_{Y}$ is.

Proof. We shall denote $\left(M, F_{\bullet}\right)_{Y}$ by $\left(M_{Y}, F\right)$. Let $\operatorname{dim} X=n, \operatorname{codim}_{X} Y=d$. Let $R=\operatorname{Gr}^{F} \mathcal{D}_{X, y}$ and $R^{\prime}=\operatorname{Gr}^{F} \mathcal{D}_{Y, y}$, and let $\mathfrak{m}$ (respectively, $\mathfrak{m}^{\prime}$ ) denote the maximal ideal in $R$ (respectively, $R^{\prime}$ ) corresponding to the origin of $T_{y}^{*} X$ (respectively, $T_{y}^{*} Y$ ). 
Both $R$ and $R^{\prime}$ are graded rings, $\operatorname{Gr}^{F} M_{y}$ and $\mathrm{Gr}^{F} M_{Y, y}$ are graded modules over them, and hence, the support of $\operatorname{Gr}^{F} M_{y}$ in $\operatorname{Spec} R$ corresponds to a homogeneous closed analytic subspace of $T^{*} U$ where $U$ is a sufficiently small open neighborhood of $y$ in $X$, and similarly for the support of $\operatorname{Gr}^{F} M_{Y, y}$ in $\operatorname{Spec} R^{\prime}$.

We need to show that $\operatorname{Gr}^{F} M_{y}$ is Cohen-Macaulay of dimension $n$ over $R$ iff $\operatorname{Gr}^{F} M_{Y, y}$ is Cohen-Macaulay of dimension $n-d$ over $R^{\prime}$. The Cohen-Macaulay property of $\mathrm{Gr}^{F} M_{y}$ is equivalent to vanishing of $\operatorname{Ext}_{R}^{j}\left(\mathrm{Gr}^{F} M_{y}, R\right)$ for $j \neq n$; as the support of $\operatorname{Ext}_{R}^{j}\left(\mathrm{Gr}^{F} M_{y}, R\right)$ is homogeneous in Spec $R$, this property holds at all points of $\operatorname{Spec} R$ iff it holds at the origin of $T_{y}^{*} X$, i.e., at the maximal ideal $\mathfrak{m}$. In other words, $\operatorname{Gr}^{F} M_{y}$ is Cohen-Macaulay of dimension $n$ over $R$ iff $\left(\operatorname{Gr}^{F} M_{y}\right)_{\mathfrak{m}}$ is Cohen-Macaulay of dimension $n$ over $R_{\mathfrak{m}}$. Similarly, $\operatorname{Gr}^{F} M_{Y, y}$ is a Cohen-Macaulay $R^{\prime}$-module of dimension $n-d$ iff $\left(\operatorname{Gr}^{F} M_{Y, y}\right)_{\mathfrak{m}^{\prime}}$ is a CohenMacaulay $R_{\mathfrak{m}^{\prime}}^{\prime}$-module of dimension $n-d$.

It follows that we need to show that $\left(\mathrm{Gr}^{F} M_{y}\right)_{\mathfrak{m}}$ is Cohen-Macaulay of dimension $n$ over $R_{\mathfrak{m}}$ iff $\left(\mathrm{Gr}^{F} M_{Y, y}\right)_{\mathfrak{m}^{\prime}}$ is Cohen-Macaulay of dimension $n-d$ over $R_{\mathfrak{m}^{\prime}}^{\prime}$.

Let $N=\operatorname{Gr}^{F} M_{Y, y}$. Let $x_{1}, \ldots, x_{n}$ be a local coordinate system in $X$ at $y$ such that $x_{1}, \ldots, x_{d}$ is a system of local equations of $Y$ in $X$. Since $Y$ is noncharacteristic, $x_{1}, \ldots, x_{d}$ is a regular $\operatorname{Gr}^{F} M_{y}$-sequence in $R$, and $N \cong$ $\operatorname{Gr}^{F} M_{y} /\left(\sum_{l=1}^{d} x_{l} \operatorname{Gr}^{F} M_{y}\right)$. Hence, $N$ is a module over $R /\left(\sum_{l=1}^{d} x_{l} R\right)$; its structure of an $R^{\prime}$-module comes from the embedding $R^{\prime} \hookrightarrow R /\left(\sum_{l=1}^{d} x_{l} R\right)$.

Let $A$ and $A^{\prime}$ be the quotients of $R /\left(\sum_{l=1}^{d} x_{l} R\right)$ and $R^{\prime}$, respectively, by the annihilators of $N$. Then $A^{\prime} \hookrightarrow A$; since $\operatorname{Gr}^{F} M_{Y}$ is $\operatorname{Gr}^{F} \mathcal{D}_{Y}$-coherent, $N$ is finite over $R^{\prime}$ and hence, $A$ is a finite $A^{\prime}$-module.

Denote by $\tilde{\mathfrak{m}}$ and $\tilde{\mathfrak{m}}^{\prime}$, respectively, the maximal ideals of $A$ and $A^{\prime}$ that correspond to the maximal ideals $\mathfrak{m}$ and $\mathfrak{m}^{\prime}$ of $R$ and $R^{\prime}$, respectively. As $A$ is a finite $A^{\prime}$-module, there are only finitely many ideals in $A$ lying over $\tilde{\mathfrak{m}}^{\prime}$, and clearly, $\tilde{\mathfrak{m}}$ is one of them. By a homogeneity argument, $\tilde{\mathfrak{m}}$ is the only ideal of $A$ lying over $\tilde{\mathfrak{m}}^{\prime}$. Hence, $A_{\tilde{\mathfrak{m}}}$ is a finite $A_{\tilde{\mathfrak{m}}^{\prime}}^{\prime}$-module.

The localization $N_{\mathfrak{m}^{\prime}}=\left(\mathrm{Gr}^{F} M_{Y, y}\right)_{\mathfrak{m}^{\prime}}$ of $N$ at $\mathfrak{m}^{\prime}$ as an $R^{\prime}$-module is the same as the localization $N_{\tilde{\mathfrak{m}}^{\prime}}$ of $N$ at $\tilde{\mathfrak{m}}^{\prime}$ as an $A^{\prime}$-module, and is isomorphic to the localization $N_{\tilde{\mathfrak{m}}}$ of $N$ at $\tilde{\mathfrak{m}}$ as an $A$-module (since $\tilde{\mathfrak{m}}$ is the only ideal of $A$ lying over $\left.\tilde{\mathfrak{m}}^{\prime}\right)$.

By [Se], Ch. IV, Proposition 12, $\operatorname{depth}_{A_{\tilde{\mathfrak{m}}^{\prime}}^{\prime}} N_{\tilde{\mathfrak{m}}^{\prime}}=\operatorname{depth}_{A_{\tilde{\mathfrak{m}}}} N_{\tilde{\mathfrak{m}}}, \operatorname{dim}_{A_{\tilde{\mathfrak{m}}^{\prime}}^{\prime}} N_{\tilde{\mathfrak{m}}^{\prime}}=$ $\operatorname{dim}_{A_{\tilde{\mathfrak{m}}}} N_{\tilde{\mathfrak{m}}}$. Clearly, depth ${ }_{R_{\mathfrak{m}^{\prime}}^{\prime}} N_{\mathfrak{m}^{\prime}}=\operatorname{depth}_{A_{\tilde{\mathfrak{m}}^{\prime}}^{\prime}} N_{\tilde{\mathfrak{m}}^{\prime}}, \operatorname{dim}_{R_{\mathfrak{m}^{\prime}}^{\prime}} N_{\mathfrak{m}^{\prime}}=\operatorname{dim}_{A_{\tilde{\mathfrak{m}}^{\prime}}^{\prime}} N_{\tilde{\mathfrak{m}}^{\prime}}$. Since $x_{1}, \ldots, x_{d}$ is a regular $\operatorname{Gr}^{F} M_{y^{-}}$-sequence in $R$, it is a regular $\left(\operatorname{Gr}^{F} M_{y}\right)_{\mathfrak{m}^{-}}$ sequence in $R_{\mathfrak{m}}$. We have $N_{\tilde{\mathfrak{m}}} \cong\left(\mathrm{Gr}^{F} M_{y}\right)_{\mathfrak{m}} /\left(\sum_{l=1}^{d} x_{l}\left(\mathrm{Gr}^{F} M_{y}\right)_{\mathfrak{m}}\right)$, and hence, $\operatorname{depth}_{A_{\tilde{\mathfrak{m}}}} N_{\tilde{\mathfrak{m}}}=\operatorname{depth}_{R_{\mathfrak{m}}}\left(\operatorname{Gr}^{F} M_{y}\right)_{\mathfrak{m}}-d, \quad \operatorname{dim}_{A_{\tilde{\mathfrak{m}}}} N_{\tilde{\mathfrak{m}}}=\operatorname{dim}_{R_{\mathfrak{m}}}\left(\operatorname{Gr}^{F} M_{y}\right)_{\mathfrak{m}}-d$.

Altogether, we see that $\operatorname{depth}_{R_{\mathfrak{m}^{\prime}}^{\prime}} N_{\mathfrak{m}^{\prime}}=\operatorname{depth}_{R_{\mathfrak{m}}}\left(\operatorname{Gr}^{F} M_{y}\right)_{\mathfrak{m}}-d$ and $\operatorname{dim}_{R_{\mathfrak{m}^{\prime}}^{\prime}} N_{\mathfrak{m}^{\prime}}=\operatorname{dim}_{R_{\mathfrak{m}}}\left(\operatorname{Gr}^{F} M_{y}\right)_{\mathfrak{m}}-\stackrel{d}{d}$. It follows that $\operatorname{depth}_{R_{\mathfrak{m}}}\left(\operatorname{Gr}^{F} M_{y}\right)_{\mathfrak{m}}=$ $\operatorname{dim}_{R_{\mathfrak{m}}}\left(\operatorname{Gr}^{F} M_{y}\right)_{\mathfrak{m}}=n$ iff depth $R_{R^{\prime}}^{\prime}, N_{\mathfrak{m}^{\prime}}=\operatorname{dim}_{R_{\mathfrak{m}^{\prime}}^{\prime}} N_{\mathfrak{m}^{\prime}}=n-d$, i.e., $\left(\operatorname{Gr}^{F} M_{y}\right)_{\mathfrak{m}}$ 
is Cohen-Macaulay of dimension $n$ over $R_{\mathfrak{m}}$ iff $N_{\mathfrak{m}^{\prime}}$ is Cohen-Macaulay of dimension $n-d$ over $R_{\mathfrak{m}^{\prime}}^{\prime}$.

Remark 2.6.3. It is not hard to see from the definitions that the functors $\mathrm{DR}_{X}$ and $\mathrm{DR}_{X}^{-1}$ are right exact in the filtered sense: if a filtered complex $\left(M^{\bullet}, F_{\bullet}\right)$ has the property that $H^{j} \operatorname{Gr}_{\bullet}^{F} M^{\bullet}=0$ for $j>j_{0}$ then $H^{j} \operatorname{Gr}_{\bullet}^{F} \operatorname{DR}_{X}\left(M^{\bullet}, F_{\bullet}\right)=0$ for $j>j_{0}$, and vice versa, and the same holds for the functor of noncharacteristic restriction $(\bullet)_{Y}$. The functor $\mathrm{DR}_{X}^{-1}$ is also left exact in the similar sense.

\section{Filtered perversity}

In this section we introduce the notion of a weakly filtered perverse differential complex; its meaning is that the complex is "locally trivial" in a certain filtered sense made precise below, and satisfies filtered cohomology vanishing conditions which are similar to the cohomology vanishing conditions for perverse sheaves.

The stratifications need to be defined only locally, which is made precise by the notion of stratified chart.

This notion of weak filtered perversity is precisely the assumption that we need to use; we call it weak because we suspect that some stronger property of "local triviality" along the strata will appear eventually.

We introduce also the notion of coherent filtered perversity which is somewhat stronger than coherence together with weak filtered perversity; we shall show in Proposition 5.2.1 that it implies holonomicity of the corresponding $\mathcal{D}_{X}$-module.

\subsection{Stratified charts.}

Definition 3.1.1. A stratified chart $\mathcal{U}$ on $X$ is the following collection of data:

(1) an open subset $U$ of $X$;

(2) an analytic stratification of $U$;

(3) for every point $x$ of any stratum $S$ of this stratification, an open neighborhood $U_{x}$ of $x$ in $U$ and an analytic submersion $\pi_{x}: U_{x} \rightarrow U_{x} \cap S$ which restricts to the identity on $U_{x} \cap S$.

3.2. The definition of filtered perversity. In what follows we denote by $Y$ the fiber $\pi_{x}^{-1}(x)$. Given a filtered differential complex $\left(\mathcal{M}^{\bullet}, F_{\bullet}\right)$ on $X$, we use the notation $\mathcal{F}_{p}^{j}=H^{j} \operatorname{Gr}_{p}^{F} \operatorname{DR}_{X}^{-1}\left(\mathcal{M}^{\bullet}, F_{\bullet}\right)$; this is a sheaf of $\mathcal{O}_{X}$-modules.

Definition 3.2.1. A filtered differential complex $\left(\mathcal{M}^{\bullet}, F_{\bullet}\right)$ on $X$ is called weakly filtered perverse if $X$ can be covered by stratified charts $\mathcal{U}$ which satisfy the following properties for every point $x$ of any stratum $S$ of $\mathcal{U}$ :

(i) for all $j$ and $p$, the sheaf $\mathcal{F}_{p}^{j}$ has the property that for all $i>0$, and for all $y \in Y$, we have $\operatorname{Tor}_{i}^{\mathcal{O}_{X, y}}\left(\mathcal{F}_{p, y}^{j}, \mathcal{O}_{Y, y}\right)=0$;

(ii) for all $j, p$, if $\mathcal{F}_{p, x}^{j} \otimes_{\mathcal{O}_{X, x}} \mathcal{O}_{Y, x}=0$ then $\mathcal{F}_{p, x}^{j}=0$;

(iii) for all $p$, all $j<0$, we have $H_{\{x\}}^{j} \operatorname{Gr}_{p}^{F} \operatorname{DR}_{Y}\left(\left(\operatorname{DR}_{X}^{-1}\left(\mathcal{M}^{\bullet}, F_{\bullet}\right)\right)_{Y}\right)=0$;

(iv) for all $p$, all $j>0$, we have $H^{j} \operatorname{Gr}_{p}^{F} \mathcal{M}^{\bullet}=0$. 
We say that $\left(\mathcal{M}^{\bullet}, F_{\bullet}\right)$ is coherent filtered perverse if it is weakly filtered perverse, the complex $\operatorname{Gr}_{p}^{F} \operatorname{DR}_{X}^{-1}\left(\mathcal{M}^{\bullet}, F_{\bullet}\right)$ has bounded $\operatorname{Gr}_{\bullet}^{F} \mathcal{D}_{Y}$-coherent cohomology, and for any point $x,\left(\mathcal{M}^{\bullet}, F_{\bullet}\right)$ is noncharacteristic with respect to $Y$.

Note that property (i) means that the complex $\operatorname{DR}_{X}^{-1}\left(\mathcal{M}^{\bullet}, F_{\bullet}\right)$ satisfies the condition (2.2), and hence, the noncharacteristic restriction $\left(\mathrm{DR}_{X}^{-1}\left(\mathcal{M}^{\bullet}, F_{\bullet}\right)\right)_{Y}$ which appears in (iii), is defined.

(We shall actually see that if $\left(\mathcal{M}^{\bullet}, F_{\bullet}\right)$ is weakly filtered perverse then $\operatorname{DR}_{X}^{-1}\left(\mathcal{M}^{\bullet}, F_{\bullet}\right)$ is isomorphic to one filtered $\mathcal{D}_{X}$-module $\left(M, F_{\bullet}\right)$, and the condition of coherent filtered perversity is equivalent to the condition that $\left(M, F_{\bullet}\right)$ coherent holonomic Cohen-Macaulay.)

3.3. Construction of stratified charts in the coherent case. Suppose that $\left(\mathcal{M}^{\bullet}, F_{\bullet}\right)$ is a filtered differential complex such that $\operatorname{Gr}_{\bullet}^{F} \mathcal{M}^{\bullet}$ has bounded coherent cohomology. Then the condition (ii) is always satisfied. We shall see here that under this assumption, there always exist stratified charts satisfying also (i).

Proposition 3.3.1. Suppose that $p: E \rightarrow X$ is a holomorphic vector bundle on $X$ and $\left\{\mathcal{F}_{i}\right\}_{I}$ is a finite collection of homogeneous coherent sheaves on $E$. Then at any point $x_{0}$ of $X$ there exists a stratified chart $\mathcal{U}$ such that for every $x \in U$ and $i \in I$ the sheaf $\left.\mathcal{F}_{i}\right|_{p^{-1} U_{x}}$ is $\left(\pi_{x} p\right)$-flat over $U_{x} \cap S$.

Proof. Let $n=\operatorname{dim} X$.

We shall construct inductively a stratified chart $\mathcal{U}^{k}$ containing $x_{0}$ such that it satisfies the required flatness property at all points $x$ of all the strata $S$ of codimension smaller than $k$.

We shall show how to construct $\mathcal{U}^{k+1}$ once $\mathcal{U}^{k}$ has been constructed. Let $U^{k}$ be the open set containing $x_{0}$ which underlies $\mathcal{U}^{k}$, and let $X^{k}$ be the union of the closures of all the strata of $\mathcal{U}^{k}$ of codimension $k$. Then $X^{k}$ is a closed analytic subset of $U^{k}$ of pure dimension $n-k$. We choose an open polydisc $\Delta^{n}$ embedded in $U^{k}$ which contains $x_{0}$ and such that there is a projection $q: \Delta^{n} \rightarrow \Delta^{n-k}$ with the property that the map $\left.q\right|_{X^{k} \cap \Delta^{n}}: X^{k} \cap \Delta^{n} \rightarrow \Delta^{n-k}$ is finite.

Consider the composite projection $q p: p^{-1} \Delta^{n} \rightarrow \Delta^{n-k}$. Let $Z$ be the set of points in $p^{-1} \Delta^{n} \subset E$ where one of the sheaves $\mathcal{F}_{i}$ is not $q p$-flat. By Frisch's theorem on the openness of the flat locus ([F], Theorem $(\mathrm{IV}, 9)$ or [BS], Theorem V.4.5), $Z$ is a closed analytic subset in $p^{-1} \Delta^{n}$ such that its image in $\Delta^{n-k}$ is negligible. All the sheaves $\mathcal{F}_{i}$ are homogeneous, and hence, $Z$ is homogeneous; it follows that $p(Z)$ is a closed analytic subset of $\Delta^{n}$. The intersection $p(Z) \cap X^{k}$ is negligible in $X^{k} \cap \Delta^{n}$ since its image under $q$ is negligible and $q$ is finite on $X^{k} \cap \Delta^{n}$; it follows that $p(Z) \cap X^{k}$ is a proper closed analytic subset of $X^{k} \cap \Delta^{n}$.

Construct $\mathcal{U}^{k+1}$ as follows. Take $U^{k+1}=\Delta^{n}$. All the strata of $\mathcal{U}^{k+1}$ of codimension less than $k$ are the intersections with $U^{k+1}$ of the strata of $\mathcal{U}^{k}$. Any $(n-k)$-stratum $S^{\prime}$ is obtained from a $(n-k)$-stratum $S$ of $\mathcal{U}^{k}$ by intersecting with $U^{k+1}$ and then removing, first, all points where $\left.q\right|_{S \cap U^{k+1}}: S \cap U^{k+1} \rightarrow \Delta^{n-k}$ is ramified, and second, the intersection with $p(Z)$. The complement of these 
strata in $U^{k+1}$ has codimension at least $k+1$; stratifying it, we complete the stratification of $\mathcal{U}^{k+1}$.

Clearly, the stata of codimension less than $k$ satisfy the required flatness condition. Let $S^{\prime}$ be any $(n-k)$-stratum constructed as above. At any point $x \in S^{\prime}$ we take a neighborhood $U_{x} \subset U^{k+1}$ in such way that $q\left(U_{x}\right)=q\left(U_{x} \cap S^{\prime}\right)$ and $q$ is an isomorphism on $U_{x} \cap S^{\prime}$. Take the projection $\pi_{x}: U_{x} \rightarrow U_{x} \cap S^{\prime}$ such that $q \pi_{x}=q$, i.e., $\pi_{x}=\left(\left.q\right|_{U_{x} \cap S^{\prime}}\right)^{-1} q$. Then all the sheaves $\mathcal{F}_{i}$ are $q p$-flat on $p^{-1}\left(U_{x} \cap S^{\prime}\right)$ since $S^{\prime}$ does not intersect $p(Z)$, and hence, they are $\pi_{x} p$-flat.

Corollary 3.3.2. Suppose that $\left(\mathcal{M}_{i}^{\bullet}, F_{\bullet}\right)$ is a finite collection of filtered differential complexes on $X$ such that $\mathrm{Gr}_{\bullet}^{F} \mathcal{M}_{i}^{\bullet}$ have bounded coherent cohomology. Then, locally at any point of $X$ there exists a stratified chart such that the properties $(i)$ and $($ ii $)$ of Definition 3.2.1 hold for each $\left(\mathcal{M}_{i}^{\bullet}, F_{\bullet}\right)$.

Proof. Consider the homogeneous coherent sheaves $\left(H^{j} \mathrm{Gr}_{\bullet}^{F} \operatorname{DR}^{-1}\left(\mathcal{M}_{i}^{\bullet}, F_{\bullet}\right)\right)^{\sim}$ on $T^{*} X$ obtained by localizing the corresponding $\operatorname{Gr}_{\bullet}^{F} \mathcal{D}_{X}$-modules.

Proposition 3.3.1 yields a stratified chart satifying the condition (i) for each $\left(\mathcal{M}_{i}^{\bullet}, F_{\bullet}\right)$; the condition (ii) is satisfied by coherence.

\section{Filtered perverse complexes correspond to filtered $\mathcal{D}$-modules}

4.1. The main theorem. Given a filtered complex $\left(M^{\bullet}, F_{\bullet}\right)$, the property that $H^{j} \operatorname{Gr}_{p}^{F} M^{\bullet}=0$ for all $p$ and all $j \neq 0$, means that $\left(M^{\bullet}, F_{\bullet}\right)$ is strict and $H^{j} M^{\bullet}=0$ for $j \neq 0$.

Another formulation of the same property is that $\left(M^{\bullet}, F_{\bullet}\right)$ is isomorphic to $H^{0}\left(M^{\bullet}, F_{\bullet}\right)$ in the filtered derived category, where $H^{0}\left(M^{\bullet}, F_{\bullet}\right)$ denotes $H^{0} M^{\bullet}$ equipped with the induced filtration.

Theorem 4.1.1. Suppose that $\left(\mathcal{M}^{\bullet}, F_{\bullet}\right)$ is weakly filtered perverse. Then, for all $p$, all $j \neq 0$,

$$
H^{j} \operatorname{Gr}_{p}^{F} \operatorname{DR}_{X}^{-1}\left(\mathcal{M}^{\bullet}, F_{\bullet}\right)=0
$$

Consequently the filtered complex $\operatorname{DR}_{X}^{-1}\left(\mathcal{M}^{\bullet}, F_{\bullet}\right)$ is strict and isomorphic in the filtered derived category to $H^{0} \mathrm{DR}_{X}^{-1}\left(\mathcal{M}^{\bullet}, F_{\bullet}\right)$ equipped with the induced filtration.

Proof. The statement is local so we may assume that $X=U$ in the definition of weak filtered perversity, $x$ lies in the stratum $S, \pi_{x}: U_{x} \rightarrow S$ is an analytic submersion which restricts to the identity on $S$, and $Y=\pi_{x}^{-1}(x)$.

We are going to show by induction on codim $S$ that the conclusion holds for the stalk of $\operatorname{Gr}_{p}^{F} \mathrm{DR}^{-1}\left(\mathcal{M}^{\bullet}, F_{\bullet}\right)$ at $x$. Thus we may assume that the conclusion holds on the complement of the stratum $S$.

Condition (iv) of Definition 3.2.1 implies that, for all $p$,

$$
H^{j} \operatorname{Gr}_{p}^{F} \operatorname{DR}_{X}^{-1}\left(\mathcal{M}^{\bullet}, F_{\bullet}\right)=0 \text { for } j>0 .
$$


By (2.3) we have

$$
H^{j} \operatorname{Gr}_{p}^{F}\left(\left(\mathrm{DR}_{X}^{-1}\left(\mathcal{M}^{\bullet}, F_{\bullet}\right)\right)_{Y}\right) \cong H^{j} \operatorname{Gr}_{p}^{F} \operatorname{DR}_{X}^{-1}\left(\mathcal{M}^{\bullet}, F_{\bullet}\right) \otimes_{\mathcal{O}_{X}} \omega_{Y / X}
$$

The induction hypothesis and (4.2) imply that

$$
\left.H^{j} \operatorname{Gr}_{p}^{F}\left(\left(\mathrm{DR}_{X}^{-1}\left(\mathcal{M}^{\bullet}, F_{\bullet}\right)\right)_{Y}\right)\right|_{Y \backslash\{x\}}=0 \text { for } j \neq 0 .
$$

Let $i:\{x\} \hookrightarrow Y$ be the embedding map. Condition (iii) of Definition 3.2.1 implies that $\mathbf{R} i^{!} \operatorname{Gr}_{p}^{F} \operatorname{DR}_{Y}\left(\left(\operatorname{DR}_{X}^{-1}\left(\mathcal{M}^{\bullet}, F_{\bullet}\right)\right)_{Y}\right)$ is acyclic in negative degrees. Here $\mathbf{R} i$ is the derived functor of the functor $i^{!}$which assigns to a sheaf $\mathcal{F}$ its sections supported in $x$; if $\mathcal{F}$ is an $\mathcal{O}_{Y}$-module or a $\mathcal{D}_{Y}$-module then $\mathbf{R} i ! \mathcal{F}$ is an an $\mathcal{O}_{Y, x}$-module or a $\mathcal{D}_{Y, x}$-module, and $\mathbf{R} i^{!}$commutes with the functors $\operatorname{Gr}_{p}^{F}$ and $\mathrm{DR}_{Y}$. Hence, $\operatorname{Gr}_{p}^{F} \mathrm{DR}_{Y} \mathbf{R} i^{!}\left(\left(\mathrm{DR}_{X}^{-1}\left(\mathcal{M}^{\bullet}, F_{\bullet}\right)\right)_{Y}\right)$ is acyclic in negative degrees; as $\mathrm{DR}_{Y}^{-1}$ is left exact (Remark 2.6.3), the complex $\operatorname{Gr}_{p}^{F} \mathbf{R} i^{!}\left(\left(\operatorname{DR}_{X}^{-1}\left(\mathcal{M}^{\bullet}, F_{\bullet}\right)\right)_{Y}\right)$ is acyclic in negative degrees, so that

$$
H_{\{x\}}^{j} \operatorname{Gr}_{p}^{F}\left(\left(\mathrm{DR}_{X}^{-1}\left(\mathcal{M}^{\bullet}, F_{\bullet}\right)\right)_{Y}\right)=0 \text { for } j<0 .
$$

Examination of the long exact sequence in cohomology associated to the inclusion $Y \backslash\{x\} \subset Y$ in the light of (4.3) and (4.4) shows that

$$
H^{j} \operatorname{Gr}_{p}^{F}\left(\left(\mathrm{DR}_{X}^{-1}\left(\mathcal{M}^{\bullet}, F_{\bullet}\right)\right)_{Y}\right)=0 \text { for } j<0 .
$$

Together with (4.2) and the condition (ii) of Definition 3.2.1 this shows that

$$
H^{j} \operatorname{Gr}_{p}^{F} \operatorname{DR}_{X}^{-1}\left(\mathcal{M}^{\bullet}, F_{\bullet}\right)_{x}=0 \text { for } j<0 .
$$

The statement of the Theorem is the combination of (4.1) and (4.5).

\section{The coherent case}

In this section we study the property of filtered perversity of a filtered differential complex $\left(\mathcal{M}^{\bullet}, F_{\bullet}\right)$ under the assumption of coherence of $H^{\bullet} \mathrm{Gr}_{\bullet}^{F} \mathcal{M}^{\bullet}$; in particular, this implies that the cohomology of $\operatorname{Gr}_{\bullet}^{F} \mathcal{M}^{\bullet}$ is bounded.

By $[\mathrm{S} 1], \quad(2.2 .10 .5)$, this is equivalent to the $\operatorname{Gr}_{\bullet}^{F} \mathcal{D}_{X}$-coherence of $H^{\bullet} \mathrm{Gr}_{\bullet}^{F} \mathrm{DR}^{-1}\left(\mathcal{M}^{\bullet}, F_{\bullet}\right)$; in case $\mathrm{DR}^{-1}\left(\mathcal{M}^{\bullet}, F_{\bullet}\right)$ is isomorphic to a single filtered $\mathcal{D}_{X}$-module, this property means that the module is $\mathcal{D}_{X}$-coherent and its filtration is good.

5.1. Duality for coherent complexes. The following technical lemma is a standard application of duality theory.

Lemma 5.1.1. Suppose that $X$ is a complex manifold of dimension $n, L^{\bullet}$ is a bounded complex of coherent $\mathcal{O}_{X}$-modules, and $x \in X$. Then for each $j$, there is a nondegenerate pairing between the spaces $H_{\{x\}}^{-j} L^{\bullet}$ and $\left(H^{j} \mathbf{R} \underline{\operatorname{Hom}}_{\mathcal{O}_{X}}\left(L^{\bullet}\right.\right.$, $\left.\left.\omega_{X}[n]\right)\right)_{x}$; the same is true for the spaces $H^{j} L_{x}^{\bullet}$ and $H_{\{x\}}^{-j} \mathbf{R} \underline{\operatorname{Hom}}_{\mathcal{O}_{X}}^{\bullet}\left(L^{\bullet}, \omega_{X}[n]\right)$. 
A nondegenerate pairing between two vector spaces is a pairing that induces a monomorphism from each of them into the (algebraic) dual of the other. (Actually, each of the vector spaces can be given a topology so that they become topologically dual. More precisely, the pairs of spaces indicated in the Lemma, are strong dual to each other with respect to certain natural FS and DFS topologies. In case the complex $L^{\bullet}$ is zero except in one degree, this statement is a particular case of a theorem of Harvey: take $K=\{x\}$ in Theorem 5.12 of [ST]. However, we do not need the topological duality; all we need is that these vector spaces are either both zero or both nonzero.)

Proof of Lemma 5.1.1 We shall establish the duality between the spaces $H_{\{x\}}^{-j} L^{\bullet}$ and $\left(H^{j} \mathbf{R} \underline{\operatorname{Hom}}_{\mathcal{O}_{X}}\left(L^{\bullet}, \omega_{X}[n]\right)\right)_{x}$; the other duality would follow by substituting the dual complex $\mathbf{R} \underline{\operatorname{Hom}}_{\mathcal{O}_{X}}\left(L^{\bullet}, \omega_{X}[n]\right)$ in place of $L^{\bullet}$.

Replacing $L^{\bullet}$ by its bounded free resolution in a neighborhood of $x$, we may assume that all the sheaves $L^{k}$ are free. Then $\mathbf{R} \underline{\operatorname{Hom}}_{\mathcal{O}_{X}}\left(L^{\bullet}, \omega_{X}[n]\right)$ is represented by $\underline{\operatorname{Hom}}_{\mathcal{O}_{X}}^{\bullet}\left(L^{\bullet}, \omega_{X}[n]\right)$, and $\left(H^{j} \mathbf{R} \underline{\operatorname{Hom}}_{\mathcal{O}_{X}}\left(L^{\bullet}, \omega_{X}[n]\right)\right)_{x} \stackrel{\sim}{=} H^{j} \operatorname{Hom}_{\mathcal{O}_{X, x}}^{\bullet}\left(L_{x}^{\bullet}\right.$, $\left.\omega_{X, x}[n]\right)$. The complex $\operatorname{Hom}_{\mathcal{O}_{X, x}}^{\bullet}\left(L_{x}^{\bullet}, \omega_{X, x}[n]\right)$ is a complex of free finitely generated $\mathcal{O}_{X, x}$-modules. Each of them has a canonical DFS topology and the differential is continuous with respect to it; moreover, the image of the differential is closed since it is closed with respect to the weaker topology of coefficientwise convergence of formal power series (Theorem 6.3.5 of $[\mathrm{H}]$ ).

Since each $L^{k}$ is free, by a theorem of Martineau ([ST], Theorem 5.9) we have that $H_{\{x\}}^{j} L^{k}$ is zero for $j \neq n$, and $H_{\{x\}}^{n} L^{k}$ can be given a natural Hausdorff FS topology in which it is a strong dual to $\operatorname{Hom}_{\mathcal{O}_{X, x}}\left(L_{x}^{k}, \omega_{X, x}\right)$; in particular, it follows that $H_{\{x\}}^{-j} L^{\bullet} \cong H^{-j-n}\left(H_{\{x\}}^{n} L\right)^{\bullet}$ where we denote $\left(H_{\{x\}}^{n} L\right) \bullet=\{\cdots \rightarrow$ $\left.H_{\{x\}}^{n} L^{k} \rightarrow H_{\{x\}}^{n} L^{k+1} \rightarrow \ldots\right\}$.

The pairing between $H_{\{x\}}^{n} L^{k}$ and $\operatorname{Hom}_{\mathcal{O}_{X, x}}\left(L_{x}^{k}, \omega_{X, x}\right)$, is given by the composition of the multiplication $H_{\{x\}}^{n} L^{k} \otimes \operatorname{Hom}_{\mathcal{O}_{X, x}}\left(L_{x}^{k}, \omega_{X, x}\right) \rightarrow H_{\{x\}}^{n} \omega_{X}$ and the residue map $H_{\{x\}}^{n} \omega_{X} \rightarrow \mathbb{C}$, and hence, the complex $\left(H_{\{x\}}^{n} L\right)^{\bullet}$ is the strong dual to the complex $\operatorname{Hom}_{\mathcal{O}_{X, x}}\left(L_{x}^{\bullet}, \omega_{X, x}[n]\right)$. As the latter is a complex of DFS spaces with Hausdorff cohomology, the former is a complex of FS spaces with Hausdorff cohomology $H^{-j-n}\left(H_{\{x\}}^{n} L\right)^{\bullet}$ strong dual to $\left(H^{j} \operatorname{Hom}_{\mathcal{O}_{X}}\left(L^{\bullet}, \omega_{X}[n]\right)\right)_{x}$. This yields a nondegenerate pairing between $H_{\{x\}}^{-j} L^{\bullet}$ and $\left(H^{j} \operatorname{Hom}_{\mathcal{O}_{X}}\left(L^{\bullet}, \omega_{X}[n]\right)\right)_{x}$.

\subsection{Holonomicity.}

Proposition 5.2.1. Suppose that $\left(\mathcal{M}^{\bullet}, F_{\bullet}\right)$ is a coherent filtered perverse complex on a complex manifold $X$. Then $\mathrm{DR}^{-1}\left(\mathcal{M}^{\bullet}, F_{\bullet}\right)$ is isomorphic in the filtered derived category to a filtered holonomic Cohen-Macauley $\mathcal{D}_{X}$-module.

Proof. The question is local and we need to prove it in a neighborhood of any point $x \in X$. 
The point $x$ is covered by a stratified $\operatorname{chart} \mathcal{U}$ satisfying properties (i)-(iv) of Definition 3.2.1; we keep the notation introduced there.

Our assumptions imply that $\operatorname{DR}_{X}^{-1}\left(\mathcal{M}^{\bullet}, F_{\bullet}\right)$ is isomorphic to a coherent filtered $\mathcal{D}_{X}$-module; we shall denote this module by $\left(M, F_{\bullet}\right)$.

We argue by induction by the codimension of the stratum $S$ containing $x$; by the inductive assumption, we may assume that $\left(M, F_{\bullet}\right)$ is holonomic CohenMacauley in the complement to $S$.

By Lemma 2.6.2, this implies that $\left(M, F_{\bullet}\right)_{Y}$ is holonomic Cohen-Macauley everywhere on $Y$ except possibly at $x$; hence, it is holonomic.

Property (iii) implies that $H_{\{x\}}^{j} \operatorname{Gr}_{p}^{F} \operatorname{DR}_{Y}\left(\left(M, F_{\bullet}\right)_{Y}\right)=0$ if $j<0$. By Lemma 5.1.1 this yields $\left(H^{j} \mathbf{R} \underline{\operatorname{Hom}}_{\mathcal{O}_{Y}}\left(\operatorname{Gr}_{p}^{F} \operatorname{DR}_{Y}\left(\left(M, F_{\bullet}\right)_{Y}\right), \omega_{Y}[\operatorname{dim} Y]\right)\right)_{x}=0$ if $j>0$. By $\S 2.5$ this implies that $H^{j} \operatorname{Gr}_{p}^{F} \operatorname{DR}_{Y} \mathbb{D}\left(\left(M, F_{\bullet}\right)_{Y}\right)=0$ if $j>0$, and by right exactness of $\mathrm{DR}_{Y}^{-1}$ we get $H^{j} \operatorname{Gr}_{p}^{F} \mathbb{D}\left(\left(M, F_{\bullet}\right)_{Y}\right)=0$ if $j>0$.

Since $\left(M, F_{\bullet}\right)_{Y}$ is a filtered $\mathcal{D}_{Y}$-module, we have $H^{j} \operatorname{Gr}_{p}^{F} \operatorname{DR}_{Y}\left(\left(M, F_{\bullet}\right)_{Y}\right)=0$ for $j>0$. By Lemma 5.1.1 and $\S 2.5$, this implies $H_{\{x\}}^{j} \operatorname{Gr}_{p}^{F} \operatorname{DR}_{Y} \mathbb{D}\left(\left(M, F_{\bullet}\right)_{Y}\right)=$ 0 for $j<0$. So we get $H_{\{x\}}^{j} \operatorname{Gr}_{p}^{F} \mathbb{D}\left(\left(M, F_{\bullet}\right)_{Y}\right)=0$ for $j<0$ by the left exactness of $\mathrm{DR}_{Y}^{-1}$. The long exact sequence of the inclusion $Y \backslash\{x\} \subset Y$ (cf. the proof of Theorem 4.1.1) yields $H^{j} \operatorname{Gr}_{p}^{F} \mathbb{D}\left(\left(M, F_{\bullet}\right)_{Y}\right)=0$ if $j<0$. (Actually, this vanishing also follows from the vanishing - see, for example, [Borel], V.2.2.2 of $\operatorname{Ext}_{\mathrm{Gr}^{F}}^{i} \mathcal{D}_{Y, x}\left(\mathrm{Gr}^{F} M_{Y, x}, \operatorname{Gr}^{F} \mathcal{D}_{Y, x}\right)$ for $i<d$ where $d$ is the codimension of the support of $\operatorname{Gr}^{F} M_{Y, x}$ in $\operatorname{Spec} \operatorname{Gr}^{F} \mathcal{D}_{Y, x}$; in our case $d=\operatorname{dim} Y$.)

Altogether, we see that $H^{j} \operatorname{Gr}_{p}^{F} \mathbb{D}\left(\left(M, F_{\bullet}\right)_{Y}\right)=0$ if $j \neq 0$, i.e., the filtered complex $\mathbb{D}\left(\left(M, F_{\bullet}\right)_{Y}\right)$ is isomorphic to one filtered module. Hence, $\left(M, F_{\bullet}\right)_{Y}$ is Cohen-Macaulay at $x$.

It follows by Lemma 2.6.2 that $\left(M, F_{\bullet}\right)$ is also Cohen-Macaulay at $x$.

\subsection{The converse to the main theorem.}

Theorem 5.3.1. If a coherent filtered $\mathcal{D}_{X}$-module $\left(M, F_{\bullet}\right)$ is holonomic and Cohen-Macauley, then $\operatorname{DR}_{X}\left(M, F_{\bullet}\right)$ is coherent filtered perverse.

Proof. Consider a point $x \in X$.

The coherence of $\left(M, F_{\bullet}\right)$ implies that $H^{\bullet} \mathrm{Gr}_{\bullet}^{F} \mathrm{DR}\left(M, F_{\bullet}\right)$ is $\mathcal{O}_{X}$-coherent. Consequently, Corollary 3.3.2 implies that in a neighborhood of $x$ there exists a stratified chart $\mathcal{U}$ such that $\operatorname{DR}\left(M, F_{\bullet}\right)$ satisfies properties (i) and (ii) with respect to it. We shall keep the notation of Definition 3.2.1.

As $M$ is holonomic, we may assume that that the characterisic variety $\mathrm{Ch}(M)$ is contained in the union of the conormal bundles to the strata of the Whitney stratification that underlies $\mathcal{U}$. It follows that the projection $\left(Y \times_{X} T^{*} X\right) \cap$ $\mathrm{Ch}(M) \rightarrow T^{*} Y$ is finite (it is even an embedding), and hence, $\left(M, F_{\bullet}\right)_{Y}$ is

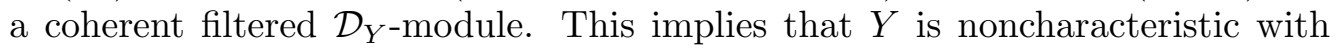
respect to $\left(M, F_{\bullet}\right)$. 
The property (iv) of Definition 3.2.1 at $x$ is satisfied by $\operatorname{DR}\left(M, F_{\bullet}\right)$ since it is satisfied by the de Rham complex of any filtered $\mathcal{D}_{X}$-module.

Let us show the property (iii) at $x$ with respect to this stratified chart.

By Lemma 2.6.2, $\left(M, F_{\bullet}\right)_{Y}$ is holonomic Cohen-Macaulay at $x$. Hence, the complex $\mathbb{D}\left(\left(M, F_{\bullet}\right)_{Y}\right)$ is isomorphic to a filtered $\mathcal{D}_{Y}$-module, and consequently, the complex $\mathrm{DR}_{Y} \mathbb{D}\left(\left(M, F_{\bullet}\right)_{Y}\right)$ satisfies (iv) at $x$ :

$$
H^{j} \mathrm{Gr}_{\bullet}^{F} \mathrm{DR}_{Y} \mathbb{D}\left(\left(M, F_{\bullet}\right)_{Y}\right)=0 \text { at } x \text { for all } j>0 .
$$

By $§ 2.5$ and Lemma 5.1.1 we get

$$
H_{\{x\}}^{j} \operatorname{Gr}_{\bullet}^{F} \operatorname{DR}_{Y}\left(\left(M, F_{\bullet}\right)_{Y}\right)=0 \text { for all } j<0 .
$$

This is the property (iii) at $x$ for the filtered complex $\operatorname{DR}_{X}\left(M, F_{\bullet}\right)$.

\section{An application to $L^{2}$ cohomology}

In this section we give an application to our results and show that in the situation of [KK2] (and under the assumption of filtered perversity of the $L^{2}$ complex) there is a local filtered isomorphism (in the sense of derived category) between the $L^{2}$ complex and the de Rham complex of the $\mathcal{D}$-module that underlies the corresponding pure Hodge module.

6.1. Let $X$ denote a Kähler manifold of dimension $n$, let $j: X^{\circ} \hookrightarrow X$ be the inclusion map of the complement of a divisor with normal crossings, and $\mathbb{E}=\left(\mathbb{E}_{\mathbb{Q}},\left(\mathcal{O}_{X^{\circ}} \otimes_{\mathbb{Q}} \mathbb{E}_{\mathbb{Q}}, F_{\bullet}\right)\right)$ a quasiunipotent polarised variation of pure Hodge structure of weight $w$ on $X^{\circ}$.

Let $\left(N, F_{\bullet}\right)$ denote the filtered $\mathcal{D}_{X}$-module underlying the polarizable Hodge module $[\mathrm{S} 1]$ which restricts to $\left(\omega_{X^{\circ}} \otimes_{\mathbb{Q}} \mathbb{E}_{\mathbb{Q}}, F_{\bullet}\right)$ on $X^{\circ}$.

Let $\left(\mathcal{A}_{(2)}^{\bullet}(\mathbb{E}), F_{\bullet}\right)$ denote the $L^{2}$-complex with coefficients in $\mathbb{E}$ constructed using the Hodge inner product in the fibers of $\mathbb{E}$ and a certain complete metric $\eta$ on $X^{\circ}$ as in [KK2], [CKS]; to keep up with our degree conventions, we shall assume that the grading of $\left(\mathcal{A}_{(2)}^{\bullet}(\mathbb{E}), F_{\bullet}\right)$ is chosen in such way that $\mathcal{A}_{(2)}^{i}(\mathbb{E})$ contains forms of degree $i+n$.

As the metric $\eta$ satisfies $\eta>C \eta_{X}$ locally in a neighborhood of any point of $X$, where $\eta_{X}$ is the metric on $X$ and $C$ a suitable positive constant, the holomorphic forms on $X$ are bounded in the pointwise norm with respect to $\eta$ and the $L^{2}$-complex is an $\mathcal{O}_{X}$-module: if $\omega$ is a section of $\mathcal{A}_{(2)}^{\bullet}(\mathbb{E})$ and $f$ is a holomorphic function then $f \omega$ is also a section of $\mathcal{A}_{(2)}^{\bullet}(\mathbb{E})$ since both $f \omega$ and $d(f \omega)=d f \wedge \omega+f d \omega$ are $L^{2}$.

We shall assume here that $\left(\mathcal{A}_{(2)}^{\bullet}(\mathbb{E}), F_{\bullet}\right)$ is weakly filtered perverse, and so is every direct summand of it (in the sense of derived category). By Theorem 4.1.1 this implies that the complex $\operatorname{DR}^{-1}\left(\mathcal{A}_{(2)}^{\bullet}(\mathbb{E}), F_{\bullet}\right)$ is strict and isomorphic in the filtered derived category to its zeroeth cohomology with the induced filtration.

By $[\mathrm{KK} 2]$ and $[\mathrm{CKS}], \mathcal{A}_{(2)}^{\bullet}(\mathbb{E})$ is isomorphic in the derived category of complexes of sheaves on $X$ to the intersection complex with coefficients in $\mathbb{E}$. We 
shall assume, moreover, that for any cross-section $Y$ appearing in the definition of weak filtered perversity, the complex $\operatorname{DR}_{Y}\left(\left(\operatorname{DR}_{X}^{-1}\left(\mathcal{A}_{(2)}^{\bullet}(X, \mathbb{E})\right)\right)_{Y}\right)$ is isomorphic to the intersection cohomology complex on $Y$ with the coefficients in $\left.\mathbb{E}\right|_{Y \cap X}$ in the derived category of complexes (without filtration). (One would even expect that the filtered complex $\operatorname{DR}_{Y}\left(\left(\operatorname{DR}_{X}^{-1}\left(\mathcal{A}_{(2)}^{\bullet}(X, \mathbb{E}), F_{\bullet}\right)\right)_{Y}\right)$ is isomorphic in the filtered derived category to $\left(\mathcal{A}_{(2)}^{\bullet}\left(Y,\left.\mathbb{E}\right|_{Y \cap X^{\circ}}\right), F_{\bullet}\right)$.)

In case $X$ is compact, both complexes of global sections $\Gamma\left(X, \mathcal{A}_{(2)}^{\bullet}(\mathbb{E})\right)$ and $\Gamma(X, \mathrm{DR}(N))$ are strict $([\mathrm{KK} 2],[\mathrm{S} 1])$, and their cohomology have pure Hodge structures. Their cohomology groups are isomorphic ([KK2]) together with the Hodge filtrations ([S2], p. 294). Here we strengthen these results and show the isomorphism at the level of sheaves (without the assumption of compactness), in the filtered derived categories:

Proposition 6.1.1. Assume that

(1) any direct summand of $\left(\mathcal{A}_{(2)}^{\bullet}(X, \mathbb{E}), F_{\bullet}\right)$ in the filtered derived category of filtered differential complexes (in particular, $\left(\mathcal{A}_{(2)}^{\bullet}(X, \mathbb{E}), F_{\bullet}\right)$ itself) is weakly filtered perverse;

(2) for any cross-section $Y$ as in Definition 3.2 .1 (weak filtered perversity), the complex $\operatorname{DR}_{Y}\left(\left(\operatorname{DR}_{X}^{-1}\left(\mathcal{A}_{(2)}^{\bullet}(X, \mathbb{E})\right)\right)_{Y}\right)$ is isomorphic to the intersection cohomology complex on $Y$ with the coefficients in $\left.\mathbb{E}\right|_{Y \cap X^{\circ}}$.

Then the filtered differential complexes $\operatorname{DR}_{X}\left(M, F_{\bullet}\right)$ and $\left(\mathcal{A}_{(2)}^{\bullet}(X, \mathbb{E}), F_{\bullet}\right)$ are isomorphic in the filtered derived category. Equivalently, the filtered $\mathcal{D}_{X}$-modules $\left(M, F_{\bullet}\right)$ and $H^{0} \mathrm{DR}^{-1}\left(\mathcal{A}_{(2)}^{\bullet}(\mathbb{E}), F_{\bullet}\right)$ are isomorphic.

Proof. By Remark 3.15 of [S2] (the idea actually going back to [KK1]), there is a direct sum decompostion in the derived category of filtered differential complexes $\left(\mathcal{A}_{(2)}^{\bullet}(\mathbb{E}), F_{\bullet}\right) \cong \mathrm{DR}_{X}\left(N, F_{\bullet}\right) \oplus\left(\mathcal{M}^{\bullet}, F_{\bullet}\right)$ and we need to show that the second summand is trivial.

Our assumptions imply that

(1) $\left(\mathcal{M}^{\bullet}, F_{\bullet}\right)$ is weakly filtered perverse, therefore, by Theorem 4.1.1, isomorphic to $\operatorname{DR}_{X}\left(M, F_{\bullet}\right)$ where $\left(M, F_{\bullet}\right)$ is a filtered $\mathcal{D}_{X}$-module;

(2) for any cross-section $Y$ as in Definition 3.2.1, the complex

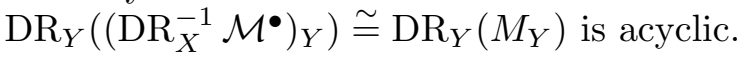

The weak filtered perversity of $\left(\mathcal{M}^{\bullet}, F_{\bullet}\right)$ implies that $X$ is covered by stratified charts satisfying properties (i)-(iv) of Definition 3.2.1. It is sufficient to show that the intersection of the support of $H^{\bullet} \mathrm{Gr}_{\bullet}^{F}\left(\mathcal{M}^{\bullet}, F_{\bullet}\right)$ with any of the charts is empty.

Assume to the contrary and consider a stratified chart and a point $x \in$ $\operatorname{Supp} H^{\bullet} \operatorname{Gr}_{\bullet}^{F}\left(\mathcal{M}^{\bullet}, F_{\bullet}\right)$ which lies on a stratum which is maximal among those which have a nonempty intersection with $\operatorname{Supp} H^{\bullet} \operatorname{Gr}_{\bullet}^{F}\left(\mathcal{M}^{\bullet}, F_{\bullet}\right)$.

Let $Y$ denote the cross-section at $x$. Then $\operatorname{Supp} M_{Y} \subseteq\{x\}$. In addition we have $H^{j} \operatorname{Gr}_{\bullet}^{F} \operatorname{DR}_{Y}\left(\left(M, F_{\bullet}\right)_{Y}\right)=0$ for $j>0$ by the right exactness of 
$\mathrm{DR}_{Y}$, and for $j<0$ by property (iii) observing that $H^{j} \operatorname{Gr}_{\bullet}^{F} \operatorname{DR}_{Y}\left(\left(M, F_{\bullet}\right)_{Y}\right) \cong$ $H_{\{x\}}^{j} \operatorname{Gr}_{\bullet}^{F} \operatorname{DR}_{Y}\left(\left(M, F_{\bullet}\right)_{Y}\right)$ since $\operatorname{Supp} \operatorname{Gr}_{\bullet}^{F} \operatorname{DR}_{Y}\left(\left(M, F_{\bullet}\right)_{Y}\right) \subseteq\{x\}$.

It follows that the filtered complex $\operatorname{DR}_{Y}\left(\left(M, F_{\bullet}\right)_{Y}\right)$ is strict. Since the complex $\operatorname{DR}_{Y}\left(M_{Y}\right)$ is acyclic it follows that the complex $\operatorname{DR}_{Y}\left(\left(M, F_{\bullet}\right)_{Y}\right)$ is filtered acyclic, and hence, $M_{Y}$ is trivial. The property (ii) of the weak filtered perversity shows that $M$ is trivial at $x$ (cf. the proof of Theorem 4.1.1), which contradicts our assumption. Hence, $M$ is trivial.

Corollary 6.1.2. In the assumptions of Proposition 6.1.1 the sheaves $H^{\bullet} \operatorname{Gr}_{\bullet}^{F} \mathcal{A}_{(2)}^{\bullet}(\mathbb{E})\left(\right.$ the $L^{2}$ - $\bar{\partial}$-cohomology, see below) are coherent.

6.2. Remarks on the $L^{2}-\bar{\partial}-$ cohomology of a singular variety. Let $\left(\mathcal{M}^{\bullet}, F_{\bullet}\right)$ be the $L^{2}$-complex of a singular subvariety $Z$ (the complex of sheaves of forms with locally summable coefficients on the nonsingular part $Z^{\circ}$ of $Z$ which are $L^{2}$ together with their differentials near all points of $Z$, both smooth and singular).

This is the sheafification of the presheaf assigning to each open set $U \subset Z$ the domain of the maximal closed extension of the differential $d$ on the Hilbert space of the $L^{2}$-forms on $U \cap Z^{\circ}$; there is another flavor of the $L^{2}$-complex constructed in a similar way by sheafification of the minimal closed extension, see details in $[\mathrm{Y}], \S 2.3$.

The complex $\mathrm{Gr}_{-p}^{F} \mathcal{M}^{\bullet}$ consists of $(p, q)$-forms $\omega^{p q}$ on $Z^{\circ}$ (for any $q$ ) which have the following properties: $\omega^{p q}$ and $\bar{\partial} \omega^{p q}$ are $L^{2}$ and moreover, there exists a form $\omega=\omega^{p q}+\omega^{p+1, q-1}+\ldots$ such that both $\omega$ and $d \omega$ are $L^{2}$.

The differential in the complex $\operatorname{Gr}_{p}^{F} \mathcal{M}^{\bullet}$ is the operator $\bar{\partial}$. Let us assume that this is a closed extension of $\bar{\partial}$. (To be precise, this means that the sections of this sheaf over an open set $U \subset Z$ form a closed extension of $\bar{\partial}$ in the Fréchet space of forms on $U \cap Z^{\circ}$ which are $L^{2}$ locally in a neighborhood of any point of $U$; the topology on this Fréchet space is given by the seminorms $\|\bullet\|_{K}$ where $K$ is a relatively compact open subset of $U$; for a form $\omega$, the value $\|\omega\|_{K}$ is the $L^{2}$ norm of $\omega$ on $K$.)

In such case the complex $\operatorname{Gr}_{p}^{F} \mathcal{M}^{\bullet}$ can be viewed as an "ideal boundary condition" (the notion due to J. Cheeger) for the operator $\bar{\partial}$ at the singularities of $Z$; this complex contains the minimal closed extension of $\bar{\partial}$ and is contained in the maximal one (their sheafifications can be defined in a way similar to those of the operator $d$ ).

If the operator $d$ on $L^{2}$ forms has the property that its minimal extension coincides with the maximal one (this is called the $L^{2}$ Stokes property $[\mathrm{C}],[\mathrm{Y}]$; it is known for conical singularities $[\mathrm{C}]$ and seems from [O3] to be a reasonable conjecture in general) then, under our assumptions, it is not hard to see that the boundary condition of $\mathrm{Gr}_{-p}^{F} \mathcal{M}^{\bullet}$ is dual to the boundary condition of $\mathrm{Gr}_{-p^{\prime}}^{F} \mathcal{M}^{\bullet}$ if $p+p^{\prime}=\operatorname{dim} Z$. (More precisely, this means the following. For any open $U \subset Z$, the dual to the Fréchet space of forms which are locally L2 on $U-$ with the topology described above - can be identified by the pairing $\langle\omega, \phi\rangle=$ 
$\int_{U \cap Z^{\circ}} \omega \wedge \phi$ with the DF space of forms $\phi$ on $U \cap Z^{\circ}$ such that the closure of $\operatorname{Supp} \phi$ in $U$ is compact. The $L^{2}$ Stokes property means that the adjoint of maximal extension of $d$ in the first space is, up to sign, the maximal extension of $d$ in the second one. The differential $\bar{\partial}$ on the sections of $\operatorname{Gr}_{-p}^{F} \mathcal{M}^{\bullet}$ on $U$ is an unbounded operator on the subspace of $(p, q)$-forms of the above Fréchet space; similarly, the differential $\bar{\partial}$ on the sections of $\mathrm{Gr}_{-p^{\prime}}^{F} \mathcal{M}^{\bullet}$ with compact support is an unbounded operator on the subspace of $\left(p^{\prime}, q\right)$-forms of the above DF space. The duality between the boundary conditions means that these two operators are adjoint up to sign.)

It is easy to see that under our assumptions, the differential in $\operatorname{Gr}_{-\operatorname{dim} Z}^{F} \mathcal{M}^{\bullet}$ is the maximal closed extension of $\bar{\partial}$; it follows by duality that the differential in $\operatorname{Gr}_{0}^{F} \mathcal{M}^{\bullet}$ is the minimal closed extension of $\bar{\partial}$.

In case the metric on $Z^{\circ}$ is complete (e.g., Saper metric), the minimal closed extension of $\bar{\partial}$ is known to coincide with the maximal one, and hence, $\operatorname{Gr}_{-p}^{F} \mathcal{M}^{\bullet}$ is just the domain of $\bar{\partial}$ with any of the boundary conditions.

In case the metric is incomplete (e.g., the restriction of the Fubini-Studi metric on the projective space to $Z^{\circ}$ ), it is known that the minimal closed extension of $\bar{\partial}$ may be different from the maximal one $[\mathrm{P}]$. The results of $[\mathrm{PS}]$ and $[\mathrm{FH}]$ suggest that in case $p=0$ the "correct" boundary condition for $\bar{\partial}$ is the minimal (Dirichlet) one, and in case $p=\operatorname{dim} Z$ the "correct" boundary condition for $\bar{\partial}$ is the maximal (Neumann) one.

This suggests that under our assumptions, the complex $\mathrm{Gr}_{-p}^{F} \mathcal{M}^{\bullet}$ is the most natural boundary condition for the operator $\bar{\partial}$, and its cohomology $H^{j} \operatorname{Gr}_{-p}^{F} \mathcal{M}^{\bullet}$ is the most natural notion of the $L^{2}-\bar{\partial}$-cohomology sheaves.

\section{Acknowledgements}

It is our pleasant duty to express our heartful thanks to all people who helped us with their advice and helpful discussions: Daniel Barlet, Alexander Beilinson, Joseph Bernstein, Jean-Luc Brylinski, Michael Kapranov, Masaki Kashiwara, David Kazhdan, Takeo Ohsawa, Claude Sabbah.

\section{References}

[BS] C. Bănică and O. Stănăşilă, Algebraic methods in the global theory of complex spaces, John Wiley \& Sons, 1976.

[Borel] A. Borel et al, Algebraic D-modules, Academic Press, 1987.

[C] J. Cheeger, On the Hodge theory of Riemannian pseudomanifolds, Proc. Symp. Pure Math. 36 (1980), 91-146.

[CGM] J. Cheeger, M. Goresky, and R. MacPherson. $L^{2}$-cohomology and intersection cohomology of singular algebraic varieties, in "Seminar on differential geometry," ed. S.-T. Yau, Princeton University Press, 1982, pp. 303-340.

[CKS] E. Cattani, A. Kaplan and W. Schmid, $L^{2}$ and intersection cohomology for a polarizable variation of Hodge structure, Invent. Math. 87 (1987), 217-252.

[FH] J. Fox and P. Haskell, Hodge decompositions and Dolbealt complexes on normal surfaces, Trans. Amer. Math. Soc. 343 (1994), 765-778. 
[F] J. Frisch, Points de platitude d'une morphisme d'espaces analytiques complexes, Invent. Math. 4 (1967), 118-138.

[GM1] M. Goresky and R. MacPherson. Intersection homology theory, Topology 19 (1980), $135-162$.

[GM2] _ Intersection homology II, Invent. Math. 71 (1983), 77-129.

[H] L. Hörmander, An introduction to complex analysis in several variables, $2^{\text {nd }}$ edition, North Holland, 1973.

[HP] W. C. Hsiang and V. Pati, $L^{2}$-cohomology of normal algebraic surfaces I, Invent. Math. 81 (1985), 395-412.

[KK1] M. Kashiwara and T. Kawai, Hodge structure and holonomic systems, Proc. Japan Acad. 62 (1986), 1-4.

[KK2] — The Poincaré lemma for a variation of Hodge structure, Publ. RIMS 23 (1987), 345-407.

[N1] M. Nagase, Remarks on the $L^{2}$-cohomology of singular algebraic surfaces, J. Math. Soc. Japan 41 (1989), 97-116.

[N2] _ Pure Hodge structure of the harmonic $L^{2}$-forms on singular algebraic surfaces, Publ. RIMS, Kyoto Univ. 24 (1988), 1005-1023.

[O1] T. Ohsawa, Cheeger-Goresky-MacPherson's conjecture for the varieties with isolated singularities, Math. Z. 206, 219-224.

[O2] _ On the $L^{2}$ cohomology groups of isolated singularities, In the volume: Advanced studies in pure mathematics 22, 1993, Prog. Diff. Geom., pp. 247-263.

[O3] - On the $L^{2}$ cohomology of complex spaces, Math. Z. 209 (1992), 519-530.

[P] W. L. Pardon, The $L^{2}-\bar{\partial}$-cohomology of an algebraic surface, Topology 28 (1989), 171-195.

[PS] W. L. Pardon and M. A. Stern, $L^{2}-\bar{\partial}-$ cohomology of complex projective varieties, J. Amer. Math. Soc. 4 (1991), 603-621.

[S1] M. Saito, Modules de Hodge polarisables, Publ. RIMS, Kyoto University 24 (1988), 849-995.

[S2] _ Mixed Hodge modules, Publ. RIMS, Kyoto University 26 (1990), 221-333.

[Sap] L. Saper, $L^{2}$ cohomology of Kähler varieties with isolated singularities, J. Diff. Geom. 36 (1992), 89-161.

[Se] J.-P. Serre, Algèbre locale, multiplicités, Lecture Notes in Mathematics 11, 3ème édition, Springer, 1975.

[ST] Y.-T. Siu, G. Trautman, Gap-sheaves and extensions of coherent analytic subsheaves, Lecture Notes in Mathematics 172, Springer-Verlag, 1971.

[Y] B. Youssin, $L^{p}$ cohomology of cones and horns, J. Diff. Geom. 39 (1994), 559-603.

[Z1] S. Zucker, Hodge theory with degenerating coefficients: $L_{2}$ cohomology in the Poincaré metric, Ann. of Math. 109 (1979), 415-476.

[Z2] _ The Hodge structures on the intersection cohomology of varieties with isolated singularities, Duke Math. J. 55, (1987), 603-616.

Dept. of Math., Pennsylvania State University, University Park, PA 16802 USA

E-mail address: bressler@math.psu.edu

Research Institute for Mathematical Sciences, Kyoto University, Kyoto, Japan

Dept. of Math. And Compuiter Science, Univ. of the Negev, Be'er Sheva', Israel E-mail address: youssin@cs.bgu.ac.il 